Conference Paper

\title{
Different Dietary Protein Sources Affect the Intestinal Tight Junctions in the Colon of Mice
}

\author{
Dina Mustika Rini ${ }^{1}$, Yunita Satya Pratiwi ${ }^{1}$, Hadi Munarko ${ }^{1}$, Takuya Suzuki ${ }^{2 *}$ \\ ${ }^{1}$ Department of Food Technology, Faculty of Engineering, Universitas Pembangunan Nasional "Veteran" Sura- \\ baya, East Java, Indonesia \\ ${ }^{2}$ Department of Biofunctional Science and Technology, Graduate School of Biosphere Science, Hiroshima Uni- \\ versity, Higashi-Hiroshima, Japan
}

*Corresponding author:

E-mail: takuya@hiroshima-u.ac.jp

\begin{abstract}
This study examined the effects of some dietary protein sources on intestinal barrier function in the colon of mice. 24 female ICR mice were randomly grouped into 4 groups $(n=6)$ fed casein, soybean protein, whey protein concentrate (WPC), and egg white diet for 28 days. In this study, we collected the colon to analyzed the TJs proteins. Cecal contents were also collected to analyze the SCFAs. Mice fed with WPC showed the highest serum LBP concentration. Soybean and casein groups had the highest expression of occludin in the colon of mice. Total SCFAs were not significantly different among the groups. Correlation analysis showed that there is a positive correlation between ZO-1 and ZO-2 with total SCFAs. There is a positive correlation between plasma LBP concentration with claudin-3 expression in the colon of mice. These results confirmed that various dietary protein intake has a significant impact on the regulation of intestinal barrier integrity and SCFAs production might be contributing to the observation.
\end{abstract}

Keywords: Short-chain fatty acid, tight junction protein, intestinal barrier, dietary protein

\section{Introduction}

The intestinal mucosal barrier is important for nutritional health, notably in immune defense and nutrient digestion. In healthy conditions, the intestinal barrier restricts the diffusion of some pathogens, toxins, microorganisms, and allergens, into the mucosal tissues and circulatory system. Several components barrier such as the adhesive mucous gel layer, Ig A, antibacterial peptides, and intercellular tight junctions (TJs) compose the intestinal barrier system. TJs has a crucial role in the physical barrier. TJs is a multiprotein complex located close at the apical portion of the transmembrane of epithelial cells. TJs consist of transmembrane proteins, including occluding (OCN), claudin (CL), and junctional adhesion molecule A (JAMA), and cytosolic proteins, like zonula occludins (ZO) proteins (Suzuki, 2020). Intestinal barrier function also sensitive to nutritional status and intestinal microbiota plays a crucial role in host health and growth (Ma et al., 2017). Furthermore, the dietary composition is one of the important factors that can affect the gut microbiota in the gut which on its own may result in multiple physiological effects (Claesson et al., 2012).

Dietary protein has short and long-term impacts in shaping the balance of gut microbiota. The effects of dietary protein on intestinal health are dependent on the sources of protein included in the diet resulting from different digestibility and specific amino acid compositions. It affects fermentation activities as well as the production of metabolites and derivative products (Ma et al., 2017). It had been reported that the composition of microbiota in the gut modulated after intake of casein and proteins isolated from plants (Day, 2013; Rist et al., 2014). Short-chain fatty acids (SCFAs) were abundantly produced in rats fed soy protein as high as SCFA-producing bacteria (Zhu et al., 2016) and casein increased the quantity of Lactobacillus and Bifidobacterium (Hongwei et al., 2011). The intestinal barrier strongly interacts

How to cite:

Rini, D. M., Pratiwi, Y. S., Munarko, H., Suzuki, T. (2020). Different dietary protein sources affect the intestinal tight junctions in the colon of mice. $1^{\text {st }}$ International Conference Eco-Innovation in Science, Engineering, and Technology. NST Proceedings. pages 264-270. doi: 10.11594/ nstp.2020.0542 
with gut microbiota and metabolites which affect specific immune responses to antigens, balancing tolerance, and effector immune responses (Takiishi et al., 2017). However, not enough data are available on how dietary proteins affect the intestinal barrier function in healthy mice. In this study, we experiment to investigate how dietary protein from casein, soybean, WPC, and egg white affect the intestinal barrier in the colon of mice.

\section{Material and Methods \\ Material and chemicals}

Rabbit primary antibodies against ZO-1, ZO-2, OCN, CL-3, CL-4, and CL-7 were purchased from Thermo Fisher Scientific. IgG (an HRP-conjugated anti-rabbit) was purchased from Sera Care. Casein was purchased from New Zealand Dairy Board, Wellington. Egg white was purchased from Taiyo Kagaku. Whey protein concentrate (WPC), soybean protein powder, and other chemicals were purchased from Wako Pure Chemical Industries.

\section{Animals}

Animals. All procedures were approved by the Animal Use Committee of the Hiroshima University and carried out according to the proper guidelines and regulations. Female ICR mice aged six weeks and weighing $\sim 27 \mathrm{~g}$ were purchased from Japan SLC and kept under conditions with controlled temperature $\left(22-24^{\circ} \mathrm{C}\right)$, humidity $(40-60 \%)$, and lighting (light: 08.00-20.00) throughout the study. The mice were acclimatized to the new environment with free access to the control diet (AIN-93G) and distilled water (ad libitum) for one week before the start of the experiment.

\section{Experimental design}

Female ICR mice aged six weeks old $(n=24)$ were adapted for one week to a standard diet based on AIN-93G before the start of the intervention. Thereafter, mice were assigned to 4 groups: casein, soybean protein, whey protein concentrate (WPC), and egg white ( $n=6 /$ group). Diet was prepared based on modified AIN-93G with $200 \mathrm{~g} / \mathrm{kg}$ egg white protein as fed based diet (Iwaya et al., 2011). Each $100 \mathrm{~g}$ diet contained $16.02 \mathrm{~g}$ protein. Bodyweight of each mouse was evaluated every day. After 28 -day feeding, all mice were sacrificed, and the blood of mice was collected from the abdominal vein which for LPS-binding protein (LBP) concentrations measurements. Colon was dissected and subjected to immunoblot analysis, while cecal contents were also collected for the determination of total SCFAs.

\section{Determination of plasma LBP}

The LBP concentration in mouse plasma was evaluated using a commercial ELISA Kit (Biometric) according to the standard protocol.

\section{Immunoblot analysis}

Colonic epithelial cells were isolated as described previously, with a few modifications. Summarily, $3 \mathrm{~cm}$ in length of proximal colonic tissues were washed with saline and cut into small pieces. Colonic segments were put into $3 \mathrm{~mL}$ isolation buffer $\left(\mathrm{Ca}^{2+-}\right.$ and $\mathrm{Mg}^{2+-}$ free HBSS supplemented with $0.1 \mathrm{mmol}$ DTT/L, $5 \mathrm{mmol}$ EDTA/L, as well as protease and phosphatase inhibitors) and keep for $1 \mathrm{~h}$ with gentle agitation and then firmly shaken to release the epithelial cells. The supernatants were centrifuged (5000 $\mathrm{rpm}, 2 \mathrm{~min}, 4^{\circ} \mathrm{C}$ ). The sediment (cell pellets) were subjected to immunoblot analysis of ZO-1, ZO-2, OCN, CL-3, CL-4, and CL-7 as explained previously (Hung \& Suzuki, 2016). Densitometric analysis of specific bands on the immunoblots was used for quantification using Image $\mathbf{J}$ software.

\section{Determination of SCFAs}

The cecal contents were homogenized in distilled water ( 9 volumes of water) then centrifuged (13000 rpm, $10 \mathrm{~min}$ at $4^{\circ} \mathrm{C}$ ). The resulting supernatant was diluted in distilled water ( 2 volumes) then deproteinized with $75 \%$ acetonitrile. Next, $20 \mu \mathrm{L}$ of $200 \mathrm{mM} 3 \mathrm{NPH}$ and $20 \mu \mathrm{L}$ of $120 \mathrm{mM}$ WSCD were added into $40 \mu \mathrm{L}$ deproteinized supernatant to derivatize the sample. Then heat at $40^{\circ} \mathrm{C}$ in a water bath for 30 min. $100 \mu \mathrm{L}$ of $100 \mu \mathrm{M}$ crotonic acid derivative was added as an internal standard. Then it was 
filtered and used for ultra-performance LC-MS to determine the organic acid concentrations.

\section{Statistical analysis}

All values are expressed as means followed by their SEMs. Statistical analysis was performed by ANOVA followed by the Tukey-Kramer post hoc test. A difference of $p<0.05$ was considered significant. Pearson analysis was used to find the correlation between two variables. SPSS (version 21.0) was used to perform the general linear model procedure.

\section{Results and Discussion}

Growth performance, food intake, and water intake

Total calories were equalized with carbohydrates and all groups fed with the same amount of protein. No differences between groups were observed in the growth performance of mice (Table 1; $p<0.05)$. The food and water intake over the experimental period were not significantly different among the groups $(p<0.05)$. All animals appeared to be healthy throughout the experiment.

Table 1. Growth performance of mice

\begin{tabular}{lrrrr}
\hline Group & Casein & Soybean & WPC & Egg white \\
\hline Body weight (0d, g) & $28.35 \pm 0.92$ & $28.15 \pm 0.47$ & $28.07 \pm 0.50$ & $28.23 \pm 0.47$ \\
Body weight (28d, g) & $35.72 \pm 1.51$ & $33.28 \pm 0.99$ & $35.08 \pm 0.99$ & $33.97 \pm 1.36$ \\
Body weight gain (g) & $7.37 \pm 0.88$ & $5.13 \pm 0.60$ & $7.02 \pm 0.76$ & $5.73 \pm 1.08$ \\
Food intake (g/day) & $4.17 \pm 0.44$ & $4.97 \pm 0.13$ & $4.08 \pm 0.48$ & $3.91 \pm 0.62$ \\
Water intake (ml/day) & $6.20 \pm 1.06$ & $9.57 \pm 1.63$ & $6.87 \pm 1.19$ & $9.31 \pm 2.00$ \\
\hline
\end{tabular}

\section{Plasma LBP concentration}

Gram-negative bacteria produced lipopolysaccharide (LPS) which known as endotoxin. LPS could upregulate LPS-binding protein (LBP) expression in the liver if it enters the circulatory system. Therefore, plasma LBP concentration is often used as a biomarker of intestinal barrier integrity (Tobias, Soldau, \& Ulevitvh, 1986).

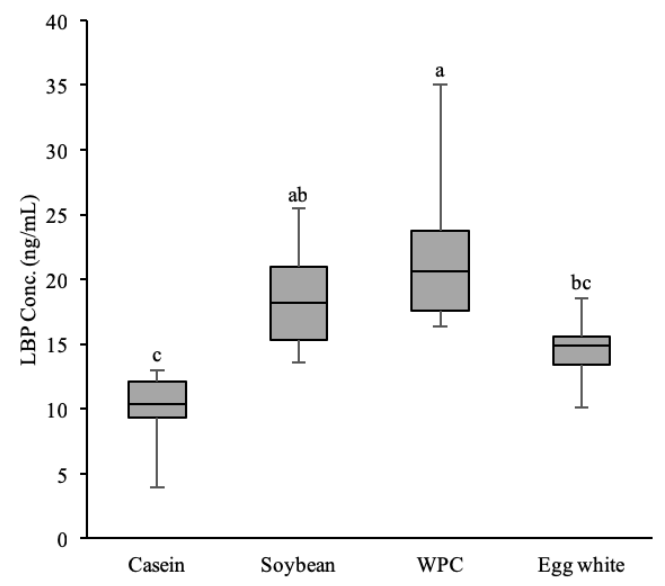

Figure 1. Plasma LBP concentration of female ICR mice fed casein, soybean, WPC, and egg white diets for 28 days. Value is means \pm SEMs, $n=6$. 


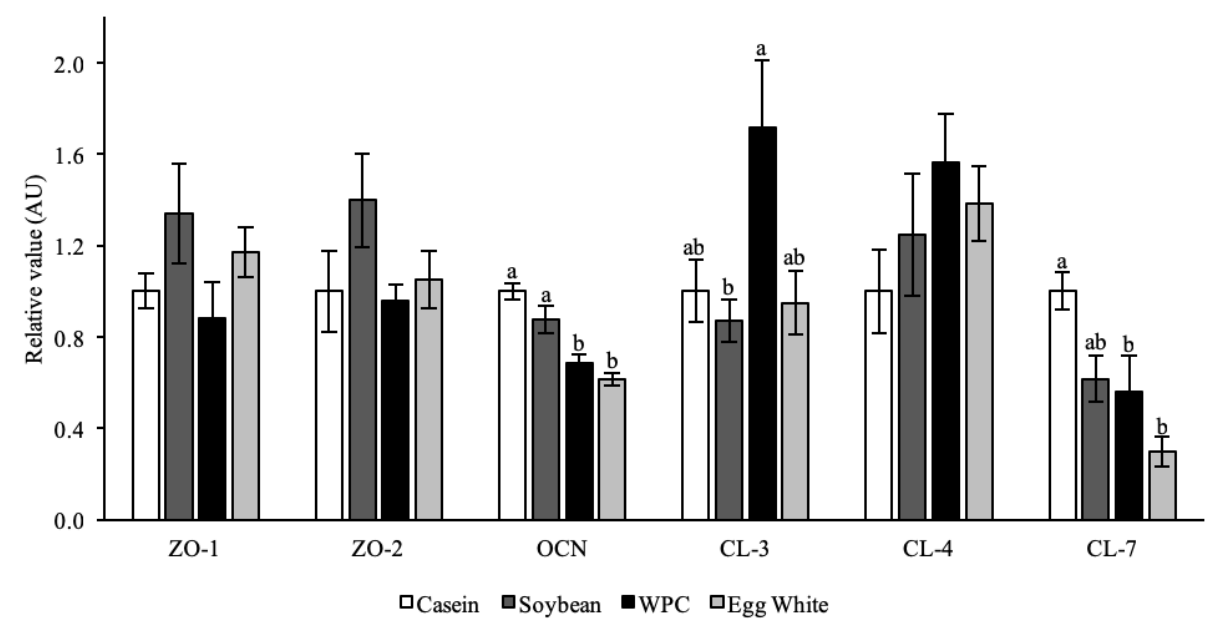

Figure 2. Expression of TJs proteins ZO-1, ZO-2, OCN, CL-3, CL-4, CL-7 in the colon of female ICR mice fed casein, soybean, WPC, and egg white diets for 28 days. Value is a means \pm SEMs, $n=6$.

The data showed that mice fed with WPC had the highest LBP concentration $(p<0.05)$ than those other groups, while the casein group showed the lowest plasma LBP concentration (Fig. $1 ; p<0.05$ ). The soybean group had a higher LBP concentration compared to the casein $(p<0.05)$ and egg white group. These results indicated that dietary protein sources affect gut-derived endotoxins level in blood serum. This may be associated with the role of intestinal tight junction in maintaining the barrier integrity which needs further investigation.

\section{Expression of tight junction proteins in colon of mice}

Expression levels of ZO-1, ZO-2, OCN, CL-3, CL-4, and CL-7 in the colon of mice was shown in Fig. 2. Casein and soybean groups showed a higher OCN expression $(p<0.05)$ than WPC and egg white group. The Casein group also showed a higher expression of CL-7 $(p<0.05)$ compared to other groups, while the soybean group was not significantly different compared to other proteins. There was no significant difference in ZO-1, ZO-2, and CL-4 expression. However, the soybean group showed the highest expression of ZO-1 and ZO-2, whereas the WPC group had the highest expression of CL-4.

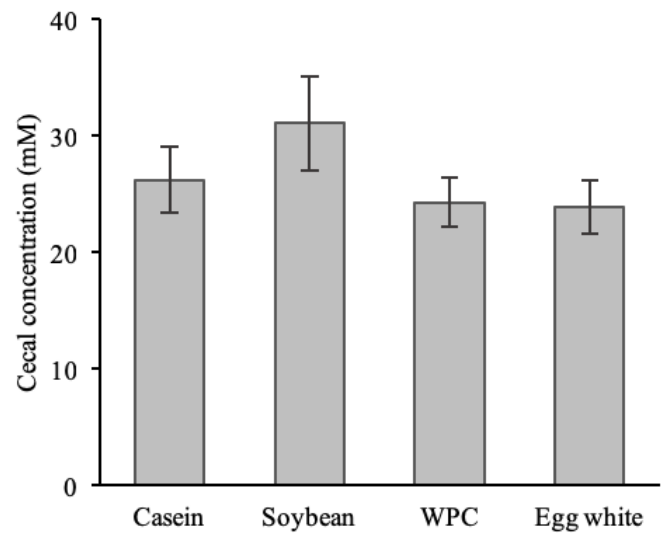

Figure 3. Total SCFAs in cecal of female ICR mice fed casein, soybean, WPC, and egg white diets for 28 days.

Value is a means \pm SEMs, $n=6$. 
Here we show that different dietary protein sources modulate TJs expression of the colon. Each TJs protein has different functional properties. Several studies have explained that claudins are the main component and the backbone of TJs. CL-3 and -4 were served as barrier-forming which has a role in decreasing paracellular permeability, whereas CL-7 was categorized as pore-forming claudins that playing roles in channel pores (increasing paracellular permeability) (Suzuki, 2013). ZO-1 binds directly to the cytoplasmic tails of claudin and occludin. Therefore, $\mathrm{ZO}-1$ has been nominated as the main player within TJs barrier function. The function of occludin is not yet fully explained, but several studies indicate that it has important roles in the TJs structure and maintain the permeability in the intestinal epithelium.

\section{Total SCFAs in cecal of mice}

Unabsorbed/undigested food components that enter into the colon were fermented by gut microbiota to generate SCFAs. In this study, we examined the total SCFAs in cecal of mice to investigate the role in regulating the intestinal barrier integrity. Figure 4 showed that different dietary protein sources did not significantly affect the total SCFAs in the cecal of mice $(p<0.05)$.

SCFAs are the main products from the fermentation activity by gut microbiota in the colon (Koh et al., 2016). The amino acid composition in various dietary protein sources may affect levels of SCFAs since different bacteria have a different substrate preference and generate different SCFAs. In the gut, some amino acids such as serine, threonine, glutamic acid, leucine, isoleucine, and arginine were metabolized by specific bacteria to produced acetic acid. Propionate can be produced from proline and threonine, while butyrate can be produced from glutamic acid, threonine, histidine, serine, and pyroglutamate (Dai et al., 2011). In this study, different dietary protein sources did not significantly affect the total SCFAs but it might affect the specific SCFAs which need further investigation.

\section{Correlation between tight junctions protein in colon with total SCFAs and plasma LBP}

Correlation analysis was done to evaluate the strength and possible relationship between two variables. To consider the role of SCFAs production in the regulation of the TJs expression and also the role of TJs in maintaining the barrier integrity, Pearson correlation coefficients between tight junction expression in the colon with plasma LBP and total SCFAs were calculated. There were positive correlations between ZO-1 and ZO-2 expression in the colon with total SCFAs $(p<0.05)$ (Table 2; Fig. 4). These observations suggest that SCFAs could be contributed to the regulation of tight junction protein in the colon of mice. Surprisingly, there was a positive correlation between claudin-3 and LBP concentration $(p<0.05)$.

Table 2. Correlation between TJs protein in colonic epithelial cells with total SCFAs

\begin{tabular}{lll}
\hline & LBP & Total SCFAs \\
\hline ZO-1 & 0.002 & $0.411^{*}$ \\
ZO-2 & 0.046 & $0.438^{*}$ \\
OCN & -0.387 & 0.133 \\
Claudin-3 & $0.503^{*}$ & -0.314 \\
Claudin-4 & 0.136 & -0.085 \\
Claudin-7 & -0.001 & 0.055 \\
\hline
\end{tabular}

$* p<0.05$ according to Pearson correlation analysis

ZO-1 and ZO-2 expression have a positive correlation with total SCFAs which might be contributed 
by acetic, propionic, and butyric acid. Butyrate is commonly known as the main energy source for colonocytes. It also protects against colorectal cancer and inflammation. Several studies persistently demonstrated that it can enhance intestinal barrier function. In the colon of rats and intestinal cells, propionic and acetic acids can increase TER and decrease permeability to Lucifer yellow in a dosedependent manner (Suzuki, Yoshida, \& Hara, 2008; Koh et al., 2016). It needs further investigation to clarify the specific SCFAs from various dietary protein sources which have a role in regulating the TJs expression in the colon of mice and their mechanisms.

Interestingly, we found that serum LBP concentration positively correlated with the expression of CL-3 in colonic epithelial cells. Claudin family commonly known as the key component and backbone of TJs and CL-3 was categorized as barrier-forming claudins which decrease paracellular permeability. This result was contradicting with the function of CL-3 to maintain the barrier function. However, intestinal mucin which secreted by goblet cells might also be responsible for the increase of serum LBP concentration, and further investigation was needed to clarify.

\section{Conclusion}

In conclusion, the expression of TJs protein in the colon of mice and plasma LBP concentration was altered by various dietary protein sources feeding. It suggests that dietary protein had a substantial influence on the modulation of intestinal barrier integrity. The SCFAs production may in part contribute to the observation although the clear mechanisms are still confused. Further investigations are needed to better understand the definite roles of dietary proteins in intestinal homeostasis.

\section{Acknowledgment}

This study was financially sponsored by Japan Society for the Promotion of Science (Grant-inAid for Young Scientists) to Takuya Suzuki.

\section{References}

Claesson, M. J. et al. (2012). Gut microbiota composition correlates with diet and health in the elderly, Nature, 488(7410), 178-184. doi: $10.1038 /$ nature 11319

Dai, Z.-L., Wu, G., \& Zhu, W-Y. (2011). Amino acid metabolism in intestinal bacteria: links between gut ecology and host health, Frontiers in Bioscience, 16(1), 1768.

Day, L. (2013). Proteins from land plants - Potential resources for human nutrition and food security, Trends in Food Science and Technology, $32(1), 25-42$.

Hongwei, Q., Xiang, Z., Han, G., Yu, B., Huang, Z., \& Chen, D. (2011). Effects of different dietary protein sources on cecum microflora in rats, African Journal of Biotechnology, 10(19), 3704-3708.

Hung, T. V., \& Suzuki, T. (2016). Dietary fermentable fiber reduces intestinal barrier defects and inflammation in colitic mice. Journal of Nutrition, 146, 1970, 6.

Iwaya, H., Kashiwaya, M., Shinoki, A., Lee, J-S., Hayashi, K., Hara, H., \& Ishizuka, S. (2011). Marginal zinc deficiency exacerbates experimental colitis induced by dextran sulfate sodium in rats, The Journal of Nutrition, 141(6), 1077-1082.

Koh, A., Vadder, F. D., Kovatcheva-Datchary, P., \& Backhed, F. (2016). From dietary fiber to host physiology: Short-chain fatty acids as key bacterial metabolites. Cell, 165(6), 1332-1345.

Ma, N., Tian, Y., Wu, Y., \& Ma, X. (2017). Contributions of the Interaction Between Dietary Protein and Gut Microbiota to Intestinal Health, Current Protein \& Peptide Science, 18(8), 1-10.

Rist, V. T. S., Weiss, E., Sauer, N., Mosenthin, R., \& Eklund, M. (2014). Effect of dietary protein supply originating from soybean meal orcasein on the intestinal microbiota of piglets, Anaerobe. Elsevier Ltd, 25, 72-79.

Suzuki, T. (2020). Regulation of the intestinal barrier by nutrients: The role of tight junctions. Animal Science Journal, 91(1), 15-21.

Suzuki, T. (2013). Regulation of intestinal epithelial permeability by tight junctions. Cellular and Molecular Life Sciences, 70(4), 631-659.

Suzuki, T., Yoshida, S., \& Hara, H. (2008). Physiological concentrations of short-chain fatty acids immediately suppress colonic epithelial permeability. British Journal of Nutrition, 100(2), 297-305. 
Takiishi, T., Fenero, C. I. M., \& Câmara, N. O. S. (2017). Intestinal barrier and gut microbiota: Shaping our immune responses throughout life, Tissue Barriers, 5(4), 1-12.

Tobias, P. S., Soldau, K., \& Ulevitch, R. J. (1986). Isolation of a lipopolysaccharide-binding acute phase reactant from rabbit serum. Journal of Experimental Medicine, 164(3), 777-793.

Zhu, Y., Lin, X., Li, H., Li, Y., Shi., X., Zhao, F., Xu, X., Li, C., \& Zhou, G. (2016). Intake of meat proteins substantially increased the relative abundance of genus Lactobacillus in rat feces. PLOS ONE, 11(4), 25-30. 